\title{
Three-Dimensional Infrared Metamaterial with Asymmetric Transmission
}

George Kenanakis, ${ }^{*}{ }^{\dagger}$ Aggelos Xomalis, ${ }^{\dagger, \dagger}$ Alexandros Selimis, ${ }^{\dagger}$ Maria Vamvakaki, ${ }^{\dagger \dagger}$ Maria Farsari, ${ }^{\dagger}$ Maria Kafesaki, ${ }^{\dagger, \ddagger}$ Costas M. Soukoulis, ${ }^{\dagger, \S}$ and Eleftherios N. Economou ${ }^{\dagger}$

${ }^{\dagger}$ Institute of Electronic Structure and Laser, Foundation for Research \& Technology-Hellas, N. Plastira 100, 70013, Heraklion, Crete, Greece

${ }^{\ddagger}$ Department of Materials Science and Technology, University of Crete, 71003 Heraklion, Crete, Greece

${ }^{\S}$ Ames Laboratory-USDOE and Department of Physics and Astronomy, Iowa State University, Ames, Iowa 50011, United States

Supporting Information

ABSTRACT: A novel three-dimensional (3D) metallic metamaterial structure with asymmetric transmission for linear polarization is demonstrated in the infrared spectral region. The structure was fabricated by direct laser writing and selective electroless silver coating, a straightforward, novel technique producing mechanically and chemically stable 3D photonic structures. The structure unit cell is composed of a pair of conductively coupled magnetic resonators, and the asymmetric transmission response results from interplay of electric and
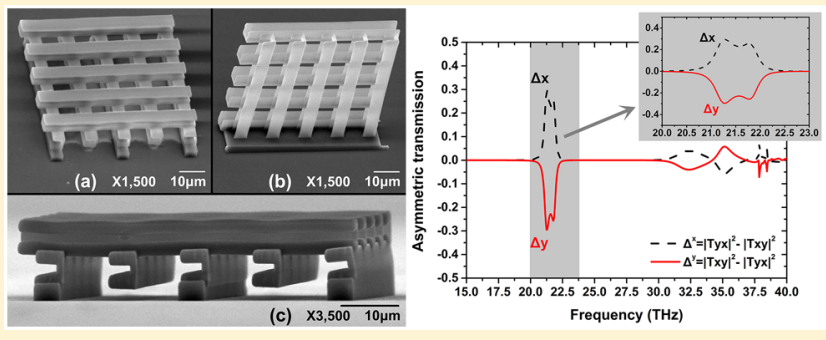
magnetic responses; this equips the structure with almost total opaqueness along one propagation direction versus satisfying transparency along the opposite one. It also offers easily adjustable impedance, $90^{\circ}$ one-way pure optical activity and backward propagation possibility, resulting thus in unique capabilities in polarization control and isolation applications. We show also that scaling down the structure can make it capable of exhibiting its asymmetric transmission and its polarization capabilities in the optical region.

KEYWORDS: metamaterials, chiral media, split-cube resonators, asymmetric transmission, direct laser writing

$\mathrm{E}$ lectromagnetic metamaterials (MMs) are artificial complex materials composed of subwavelength-scale building blocks and having electromagnetic (EM) properties beyond anything that can be found in nature; these properties are due mainly to the geometric structure of the MM building blocks (often called "meta-atoms") and less due to their constituent materials. Adjusting properly the MMs' geometrical features, one can achieve properties such as negative refractive index, magnetism at optical frequencies, perfect absorption, and enhanced optical nonlinearities. Driven by these properties, several applications of two- dimensional (2D) and threedimensional (3D) metamaterials have been proposed, including subwavelength resolution imaging systems, compact polarization control elements, cloaking devices, filters, absorbers, sensors, and biosensors. ${ }^{1-5}$

An unusual and counterintuitive phenomenon that becomes possible with metamaterials and has attracted recently considerable attention is that of asymmetric transmission (AT), i.e., different transmission of a polarized wave if sent by the two opposite sides of a metamaterial slab. This diodelike response, which originates from the different wavestructure coupling if the wave originates from the two opposite sides of the slab, does not violate Lorenz's reciprocity theorem and can find use in many EM wave manipulation devices such as polarization rotators, ${ }^{6}$ isolators, and circulators. ${ }^{7}$
The possibility of asymmetric transmission in reciprocal structures was discussed first in the context of $2 \mathrm{D}$ planar chiral metamaterials, where asymmetric transmission for circularly polarized waves was observed. ${ }^{8-11}$ This asymmetric transmission originates from the fact that an incident circularly polarized wave sees different handedness for the two opposite propagation directions. Many 2D planar metamaterials with asymmetric transmission for circularly polarized waves have been proposed and discussed in the literature, including microwave and $\mathrm{THz}$ chiral structures, ${ }^{8,9,12}$ chiral plasmonic metamolecules, $^{13}$ and general anisotropic 2D structures. ${ }^{14}$ These structures though, due to their $2 \mathrm{D}$ character, which implies mirror symmetry along the normal to their plane (propagation) direction, do not show asymmetric transmission for linearly polarized waves, ${ }^{10}$ restricting thus the possibilities of the asymmetric transmission effect in applications.

To achieve asymmetric transmission for linearly polarized waves, one needs to break the mirror symmetry also along the propagation direction, avoiding simultaneously any $C_{4}$ rotational symmetry in the structure plane (considering it normal to the propagation direction). ${ }^{15}$ Recently, employing such anisotropic metamolecules, various metamaterial structures

Received: October 14, 2014

Published: January 5, 2015 

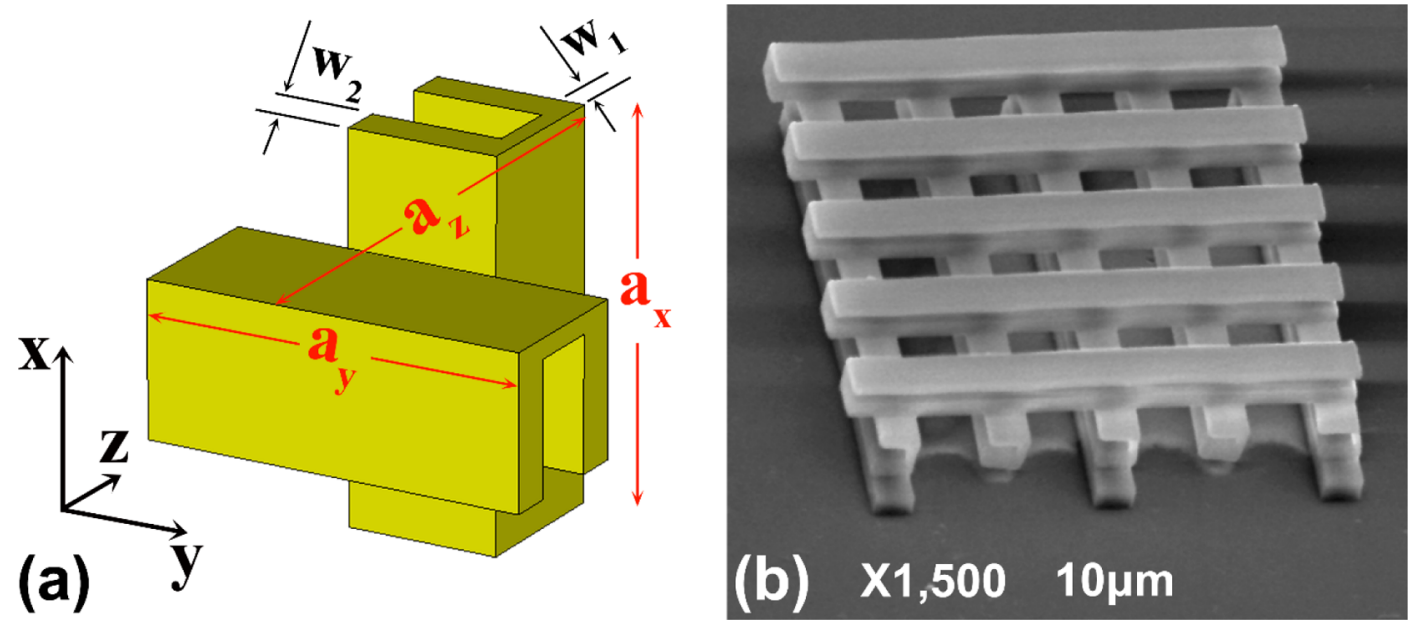

Figure 1. (a) Schematic of the unit cell of the 3D SCR metamaterials under consideration. The dimensions of the fabricated structure are $a_{x}=a_{y}=$ $8.0 \mu \mathrm{m}, a_{z}=9.1 \mu \mathrm{m}, w_{1}=600 \mathrm{~nm}$, and $w_{2}=850 \mathrm{~nm}$, respectively. The wave propagation is along the $z$-direction. (b) Top view of the SEM image of the $3 \mathrm{D}$ SCR metamaterials under consideration, recorded at $15 \mathrm{kV}$. The magnification scale can be seen below the SEM image.

showing asymmetric transmission for linearly polarized waves have been proposed and demonstrated, and the conditions relating asymmetric transmission and structure asymmetries have been discussed in detail. ${ }^{15-19}$

Most of the AT structures demonstrated to date are structures based on the planar bilayer conductor configuration; that is, the unit cell is formed by a pair of planar conductors, electromagnetically coupled. Transmission asymmetry originates from the asymmetric coupling of electric dipolar resonant modes of the two planar conductors. Despite the ease of fabrication, using such planar structures limits the possibilities offered by fully $3 \mathrm{D}$ volumetric structures, which allow a much larger variety of structure designs, allowing much more subwavelength-scale metamaterial resonators and larger flexibility in the coupling between adjacent resonators; not only electromagnetic coupling but coupling via electric-current connections is allowed. Moreover, they allow asymmetric transmission to be generated due to asymmetric coupling of magnetic rather than electric dipole resonances.

The involvement of magnetic resonators, besides electric resonators, in the AT effect offers the potential to adjust at will the structure impedance, matching for example the free space impedance and achieving higher transmission values. Moreover, it offers the potential to combine AT with backward propagation, allowing, for example, backward propagation along one direction versus forward propagation in the opposite direction, leading to a peculiar structure response.

In this work we demonstrate asymmetric transmission generated by magnetic resonance coupling in a $3 D$ volumetric structure based on two perpendicular split-cube resonators (SCRs); see Figure 1. The structure was fabricated by direct laser writing $(\mathrm{DLW})^{20,21}$ and selective silver coating, ${ }^{22,23}$ a technique able to provide fully $3 \mathrm{D}$ structures with deepsubwavelength resolution. It operates in the middle infrared $(15-40 \mathrm{THz})$, a frequency region of significant technological interest, where the requirement for EM wave control components is still high, despite substantial recent developments. Our structure, which exhibits asymmetric transmission for linear polarization only (and not for circular), while it is quite transparent along one propagation direction, shows almost total isolation along the opposite direction, providing unique possibilities in polarization isolation applications. To achieve such an isolation response (zero vs high transmission), both the electric and magnetic responses of the split-cube resonator components of the structure were exploited. This involvement of both electric and magnetic resonances equips the structure also with the possibility of one-way $90^{\circ}$ pure optical activity, offering additional capabilities for the control of the light polarization. ${ }^{4,24}$

In what follows we present the structure design (Section 2), the fabrication (Section 3, detailed in the Supporting Information), and the methods employed to analyze the electromagnetic response of the structure, both theoretically and experimentally (Section 4). In Section 5 we present the results of the electromagnetic characterization of the structure, which include reflection measurements and simulations combined with transmission simulation and analysis, followed by a detailed discussion of our structure capabilities. Finally, in Section 6 we discuss a miniaturized, nanometer-scale version of the structure, which offers optical (at $750 \mathrm{~nm}$ wavelength) asymmetric transmission for linearly polarized light. The paper ends with our conclusions.

\section{THE DESIGN}

The unit cell of the $3 \mathrm{D}$ metamaterial design employed in the present study is shown in Figure 1. It consists of two split-cube resonator structures rotated by $90^{\circ}$ with respect to each other along the $z$ (propagation)-direction. The geometrical structure parameters for the structure as fabricated and characterized experimentally are detailed in the caption of Figure 1.

As can be seen, the structure lacks mirror symmetry along the $x$-, $y$-, and $z$-directions as well as $C_{4}$ rotational symmetry in the $x-y$ plane. It consists of two conductively coupled identical magnetic resonators with the back one (along $z$ ) rotated $90^{\circ}$ with respect to the front one. According to the literature, ${ }^{17,18}$ structures of this symmetry are expected to allow asymmetric transmission for linearly polarized waves only. This comes from asymmetric cross-polarization conversion of a linearly polarized wave incident on the two opposite sides of the slab (along the $z$-direction) and depends strongly on the polarization of the incident wave in the $x-y$ plane. 


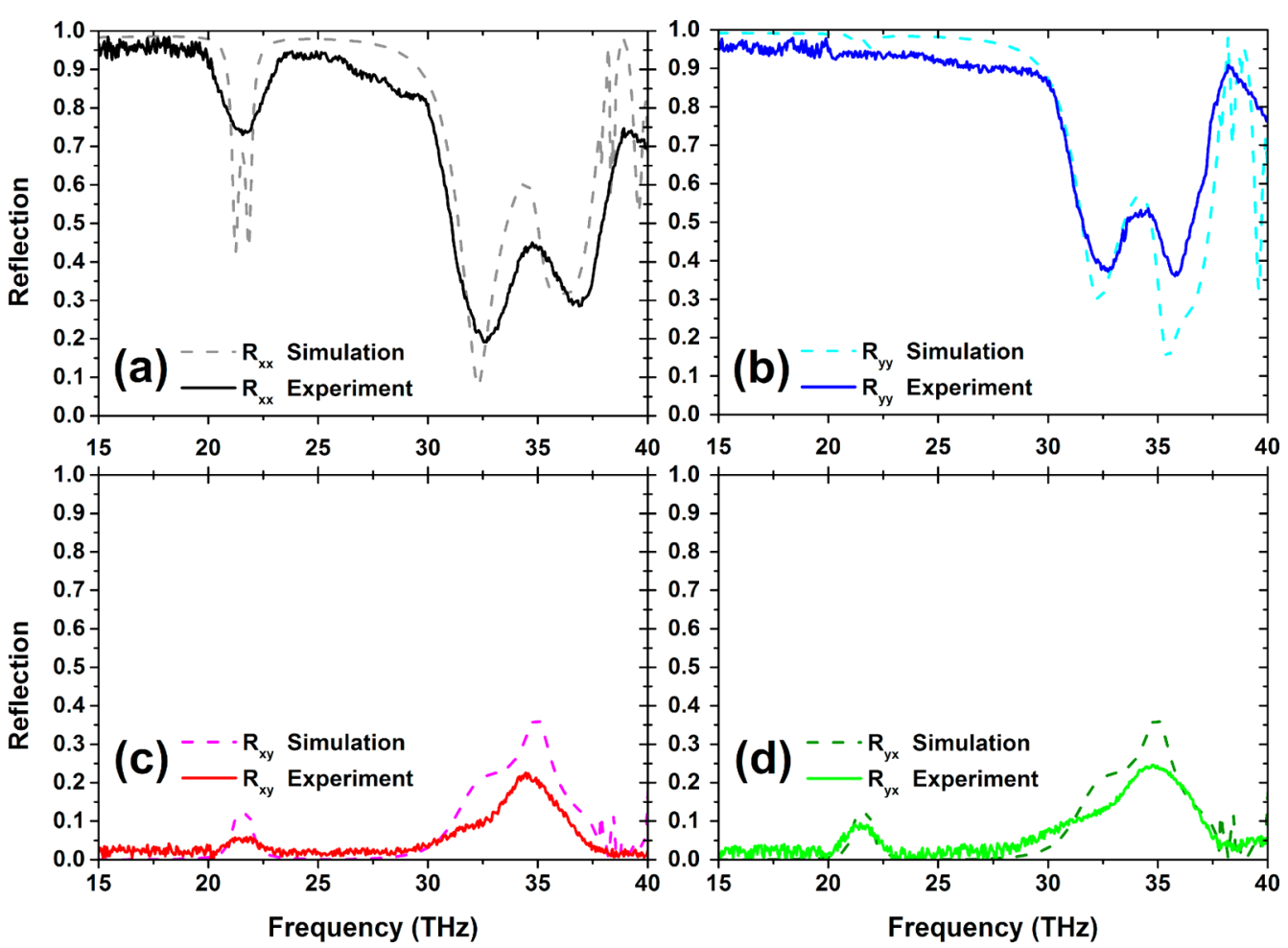

Figure 2. Simulated (dashed lines) and measured (solid lines) magnitude of the reflection components for the 3D SCR metamaterials under consideration and for a linearly polarized incident wave. All four reflection components, $R_{x x}, R_{y y}, R_{x y}$, and $R_{y x}$, can be seen in (a), (b), (c), and (d), respectively.

\section{STRUCTURE FABRICATION}

As was mentioned in the introduction, our structure was fabricated by direct laser writing followed by electroless silver plating. DLW by multiphoton polymerization is a $3 \mathrm{D}$ printing technology that allows the fabrication of $3 \mathrm{D}$ structures with resolution below $100 \mathrm{~nm}$. Briefly, the beam of an ultrafast laser is tightly focused inside the volume of a transparent and photosensitive monomer, causing it to absorb two or more photons and polymerize locally. By moving the beam in three dimensions inside the photopolymer volume, one can fabricate $3 \mathrm{D}$ structures of great accuracy. The photosensitive material used for the fabrication is an organic-inorganic composite, produced by the addition of methacryloxypropyl trimethoxysilane (MAPTMS) to zirconium n-propoxide. 2(Dimethylamino)ethyl methacrylate (DMAEMA), acting as a metal-binding moiety, was also added and copolymerized with MAPTMS upon photopolymerization. Michler's ketone (4,4bis(diethylamino)benzophenone, BIS) was used as the photoinitiator. Further information on the fabrication technique and the photosensitive material synthesis can be found in the Supporting Information.

\section{ELECTROMAGNETIC CHARACTERIZATION}

The experimental EM characterization of the structure discussed here was performed in the frequency region 15-40 $\mathrm{THz}$, through reflection measurements, performed using a Bruker Vertex 70v Fourier-transform infrared spectrometer with a collimated beam, attached to a Bruker Hyperion 2000 infrared microscope and two linear $\mathrm{ZnSe}$ grid polarizers.

The results of the measurements were compared in all cases with corresponding reflection simulations. For these, we used a commercial three-dimensional full-wave solver (CST Microwave Studio, Computer Simulation Technology $\mathrm{GmbH}$,
Darmstadt, Germany) based on the finite element method. We considered in the simulations a single unit cell, as shown in Figure 1, with periodic boundary conditions along the $x$ - and $y$ directions, while an incident plane wave propagating along the $z$-direction was used to excite the structure. For modeling the metallic parts of the structure (silver; yellow color in Figure 1) we considered a lossy-metal model with a conductivity of $\sigma_{\mathrm{Ag}}=$ $5.71 \times 10^{6} \mathrm{~S} / \mathrm{m}$, in agreement with previous conductivity measurements. ${ }^{22}$

\section{RESULTS AND DISCUSSION}

Reflection Measurements. Since the 3D SCR structure was fabricated on glass, which is not transparent in the farinfrared region of interest, we characterized the structure experimentally by measuring the reflection rather than the transmission coefficients. The transmission coefficients were concluded indirectly, through simulations. Thus, for a linearly polarized incident wave four reflection components were measured, $R_{x x}, R_{x y}, R_{y x}$, and $R_{y y}$, where the first and second lower indices indicate the output and input signal polarizations, respectively, e.g., $R_{x y}=E_{x}^{\mathrm{r}} / E_{y}^{\mathrm{i}}$, where $E_{y}^{\mathrm{i}}$ is the incident $y$ polarized electric field and $E_{x}^{\mathrm{r}}$ is the reflected $x$-polarized electric field. $^{25}$

In Figure $2 \mathrm{a}-\mathrm{d}$ we present both the experimental (solid lines) and the simulated (dashed lines) copolarized and crosspolarized reflection amplitudes $\left(\left|R_{x x}\right|,\left|R_{y y}\right|\right.$ and $\left|R_{x y}\right|,\left|R_{y x}\right|$, respectively) for the $3 \mathrm{D}$ SCR structure under consideration. One can observe in Figure 2 the very good quantitative agreement between simulations and experiments, indicating the very good quality of the fabrication of the samples (slight disagreements in a few cases are probably due to the better resolution in the simulation compared to the experiment and to the slight sample roughness and imperfections; this is the origin 


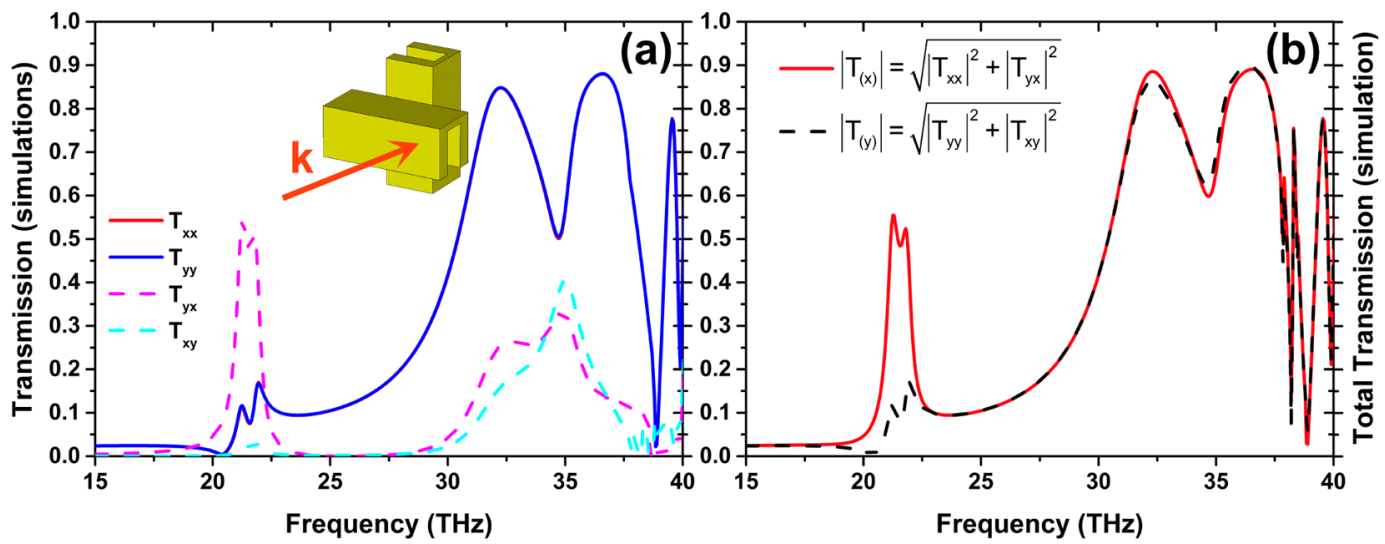

Figure 3. (a) Simulated transmission components (absolute values) for linearly polarized incident waves propagating through the 3D SCR structure along the forward (positive) $z$-direction. The red solid curve coincides with the blue solid curve. (b) Total transmitted amplitude for $x$ - and $y$ polarized incident wave, $\left|T_{(x)}^{f}\right|$ and $\left|T_{(y)}^{f}\right|$, respectively, propagating in the forward $z$-direction. Note that for our structure $\left|T_{(y)}^{f}\right|=\left|T_{(x)}^{b}\right|$; thus the asymmetric transmission response of the structure for an $x$-polarized incident wave is demonstrated.

also of the not-well-resolved experimental double dip of simulated $R_{x x}$ around $21.5 \mathrm{THz}$; the double character of the dip is due to the coupling (leading to splitting) of the magnetic resonant modes of the two SCRs of the unit cell, as we will discuss later). This very good agreement between simulations and experiments also allows us to base the subsequent calculations and analysis on the simulation results.

Transmission Properties: Asymmetric Transmission. In order to study wave propagation and transmission in anisotropic structures like the one discussed here, usually the transmission matrix is employed, $\stackrel{\leftrightarrow}{\mathbf{T}}$, which connects the transmitted with the incident fields as follows:

$$
\left(\begin{array}{c}
E_{x}^{\mathrm{t}} \\
E_{y}^{\mathrm{t}}
\end{array}\right)=\left(\begin{array}{cc}
T_{x x} & T_{x y} \\
T_{y x} & T_{y y}
\end{array}\right)\left(\begin{array}{c}
E_{x}^{\mathrm{i}} \\
E_{y}^{\mathrm{i}}
\end{array}\right)
$$

In eq 1 propagation of an incident plane wave along the $z$ direction is considered, and $E_{x}^{\mathrm{t}}, E_{y}^{\mathrm{t}}$ denote the $x$ and $y$ components of the transmitted electric field, respectively.

By definition, asymmetric transmission, often symbolized as $\Delta$, is the difference in the transmittance (transmitted intensity divided by the incident intensity) for waves propagating along two opposite directions (in our case the forward (positive) and backward (negative) $z$-direction), i.e.,

$$
\Delta=\left|\mathbf{T}^{\mathrm{f}}\right|^{2}-\left|\mathbf{T}^{\mathrm{b}}\right|^{2}
$$

with $|\mathbf{T}|^{2}=\left|\mathbf{E}^{\mathrm{t}}\right|^{2} /\left|\mathbf{E}^{\mathrm{i}}\right|^{2}=\left(\left|E_{x}^{\mathrm{t}}\right|^{2}+\left|E_{y}^{\mathrm{t}}\right|^{2}\right) /\left|\mathbf{E}^{\mathrm{i}}\right|^{2}$, where the superscripts $\mathrm{f}$ and $\mathrm{b}$ denote the forward and backward $z$ direction, respectively.

Let us consider the incident wave to be a linearly polarized wave polarized along the $x$-direction, i.e., $E_{y}^{\mathrm{i}}=0$. The transmitted wave then will have in general both $x$ and $y$ components, and the normalized transmitted intensity will be I $\left.\mathbf{T}\right|^{2}=\left|\mathbf{T}_{(x)}\right|^{2}=\left|T_{x x}\right|^{2}+\left|T_{y x}\right|^{2}$. (For a $y$-polarized incident wave it will be $|\mathbf{T}|^{2}=\left|\mathbf{T}_{(y)}\right|^{2}=\left|T_{y y}\right|^{2}+\left|T_{x y}\right|^{2}$.)

In systems containing only reciprocal materials, like the one studied here, the transmission matrix for the backward propagation direction is connected to the transmission matrix for the forward direction as follows: ${ }^{15}$

$$
\left(\begin{array}{cc}
T_{x x}^{\mathrm{b}} & T_{x y}^{\mathrm{b}} \\
T_{y x}^{\mathrm{b}} & T_{y y}^{\mathrm{b}}
\end{array}\right)=\left(\begin{array}{cc}
T_{x x}^{\mathrm{f}} & -T_{y x}^{\mathrm{f}} \\
-T_{x y}^{\mathrm{f}} & T_{y y}^{\mathrm{f}}
\end{array}\right)
$$

Thus, the asymmetric transmission for an $x$-polarized incident wave becomes

$$
\begin{aligned}
\Delta & =\Delta^{(x)} \\
& =\left|T_{x x}^{\mathrm{f}}\right|^{2}+\left|T_{y x}^{\mathrm{f}}\right|^{2}-\left|T_{x x}^{\mathrm{b}}\right|^{2}-\left|T_{y x}^{\mathrm{b}}\right|^{2} \\
& =\left|T_{y x}^{\mathrm{f}}\right|^{2}-\left|T_{x y}^{\mathrm{f}}\right|^{2}
\end{aligned}
$$

(For a $y$-polarized incident wave, $\Delta=\Delta^{(y)}=\left|T_{x y}^{\mathrm{f}}\right|^{2}+\left|T_{y y}^{\mathrm{f}}\right|^{2}-1$ $\left.T_{x y}^{\mathrm{b}}\right|^{2}-\left|T_{y y}^{\mathrm{b}}\right|^{2}=\left|T_{x y}^{\mathrm{f}}\right|^{2}-\left|T_{y x}^{\mathrm{f}}\right|^{2}=-\Delta^{(x)}$.)

Equation 4 shows that, in the case of reciprocal systems, asymmetric transmission is in fact asymmetric cross-polarization conversion between two perpendicular incident wave polarizations. This shows that to evaluate the asymmetric transmission, instead of performing a transmission/reflection experiment (or simulation) for two opposite propagation directions, one can realize the experiment/simulation only for the forward direction and for both $x$ - and $y$-polarized incident waves.

Following this approach, in Figure $3 \mathrm{a}$ we present the simulated transmission components $T_{x x}, T_{y y}, T_{x y}, T_{y x}$ (absolute values) for waves propagating along the positive $z$-direction in the 3D SCR structure; in Figure $3 \mathrm{~b}$ we show the total transmitted field amplitude for $x$ and $y$ incident wave polarizations, $\left|\mathbf{T}_{(x)}\right|=\left(\left|T_{x x}\right|^{2}+\left|T_{y x}\right|^{2}\right)^{1 / 2}$ and $\left|\mathbf{T}_{(y)}\right|=\left(\left|T_{y y}\right|^{2}+\mid\right.$ $\left.\left.T_{x y}\right|^{2}\right)^{1 / 2}$, respectively. As can be seen in Figure $3 \mathrm{a}$, in our case $T_{x x}=T_{y y}$ in all frequency regions; this can be concluded also by observing the symmetry of the structure. On the other hand the cross-polarized transmission amplitudes $T_{y x}$ and $T_{x y}$ are quite different, especially in the region centered at $21.5 \mathrm{THz}$, indicating strong asymmetric transmission. This strong asymmetric transmission for frequencies between 21 and 22 $\mathrm{THz}$ is demonstrated clearly in Figure $3 \mathrm{~b}$, where the total transmission is shown (note that due to the equality of copolarized transmission amplitudes $T_{x x}$ and $T_{y y}$, the total transmission for a $y$-polarized incident wave propagating along the positive $z$-direction is equal to the total transmission of an $x$-polarized incident wave propagating in the negative $z$ direction, i.e., $\left.\left|\mathbf{T}_{(y)}^{\mathrm{f}}\right|=\left|\mathbf{T}_{(x)}^{\mathrm{b}}\right|\right)$. The strong asymmetric transmission of the structure is highlighted further in Figure 4, where the parameter $\Delta$ is plotted, according to eq 4 .

Observing the results of Figure $3 b$, one should note that, contrary to most of the structures discussed so far in the literature, the asymmetric transmission band here at around 


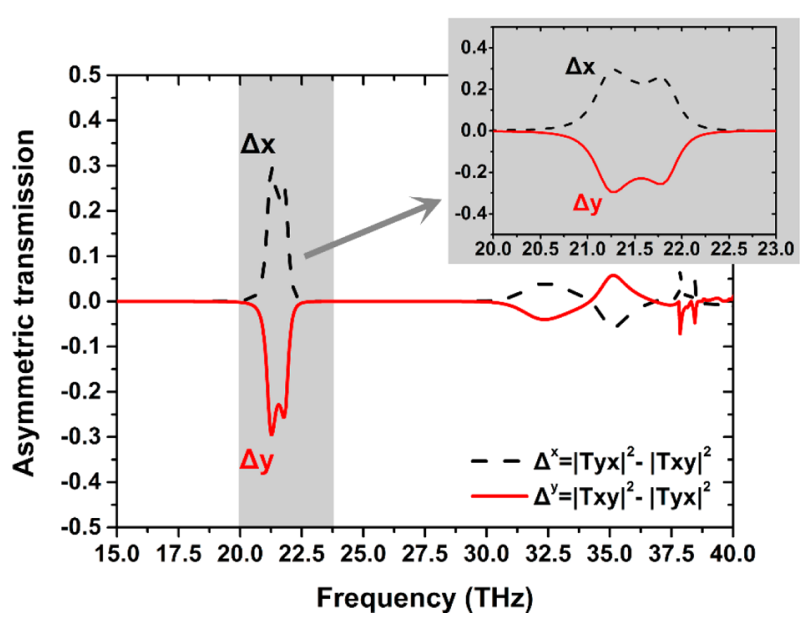

Figure 4. Simulated AT parameter $\Delta$ for the 3D SCR metamaterial under consideration. Black dashed and red solid lines correspond to AT of $x$ - and $y$-linearly polarized incident waves propagating in the forward $z$-direction. The asymmetry factor curves show a quite broadband peak with asymmetric transmission close to $30 \%$, centered at about $21.5 \mathrm{THz}$ (see inset), and two peaks of smaller asymmetric transmission, at around 32.5 and $35.1 \mathrm{THz}$, respectively.

21.5 $\mathrm{THz}$ appears as a pass-band imposed in a broad region of forbidden propagation and transmission (from 0 to $\sim 28 \mathrm{THz}$ ). This broad stop-band is due to a negative permittivity response provided by the metallic cubes that are parallel to the incident electric field direction (the metallic cubes act as a wire-grid polarizer), resulting in small copolarized transmission amplitudes $\left|T_{x x}\right|$ and $\left|T_{y y}\right|$, as shown in Figure $3 a$. The transmission band around $21.5 \mathrm{THz}$ (i.e., the asymmetric transmission band) is due to a magnetic resonance of the parallel to the external magnetic field cubes, coupled to the equivalent magnetic resonance of the perpendicular cubes, and superimposed to the negative permittivity response of the cubes, as we will demonstrate later on. (The double-peak character of that band is a result of the mode-splitting due to this magnetic resonance coupling.)

From the results of Figure $3 \mathrm{~b}$ one can see also that in the asymmetric transmission band around 21.5 the structure looks quite transparent along one propagation direction and quite opaque if "seen" from the opposite direction (for a linearly polarized wave polarized along one of the principal lattice directions). This "zero" versus "large" transmittance (which results from the large difference between $\left|T_{x y}\right|$ and $\left|T_{y x}\right|$ combined with the small values of the copolarized transmission amplitudes $\left|T_{x x}\right|$ and $\left|T_{y y}\right|^{26}$ ) is a highly desired feature in polarization control applications.

As was mentioned also earlier, from the results of Figure 3a one can notice that for our structure $T_{x x}=T_{y y}$. This results in the asymmetric transmission occurring for only linearly polarized waves, while the transmission for circularly polarized incident waves is fully symmetric, as shown in the literature. ${ }^{15,17}$ The asymmetric transmission for linearly polarized waves in this case is a function of the polarization angle, $\varphi$, according to the relation $\Delta^{(x)}=\left(\left|T_{y x}\right|^{2}-\left|T_{x y}\right|^{2}\right) \cos (2 \varphi){ }^{15}$

The asymmetric transmission response of our structure can be also indicated, but not definitively concluded, from the reflection measurements and simulations presented in Figure 2. Taking into account that due to the symmetry of the structure I $\left.\mathbf{T}_{(x)}^{\mathrm{b}}\right|^{2}=\left|\mathbf{T}_{(y)}^{\mathrm{f}}\right|^{2}$ and $\left|\mathbf{R}_{(x)}^{\mathrm{b}}\right|^{2}=\left|\mathbf{R}_{(y)}^{\mathrm{f}}\right|^{2}$, where $\left|\mathbf{R}_{(y)}^{\mathrm{f}}\right|^{2}\left(\left|\mathbf{R}_{(x)}^{\mathrm{b}}\right|^{2}\right)$ is the reflectance of a forward (backward) propagating incident wave polarized along the $y$-direction ( $x$-direction), and $\left|\mathbf{R}_{(x)}\right|^{2}=\left|R_{x x}\right|^{2}$ $+\left|R_{y x}\right|^{2},\left|\mathbf{R}_{(y)}\right|^{2}=\left|R_{y y}\right|^{2}+\left|R_{x y}\right|^{2}$ (see Figure 2 for the reflection components), one can see that in the absence of absorption the asymmetric transmission formula of eq 4 can become $\Delta^{(x)}=$ । $\left.T_{y x}\right|^{2}-\left|T_{x y}\right|^{2}=\left|R_{y y}\right|^{2}-\left|R_{x x}\right|^{2}$ (here the superscripts $\mathrm{f}, \mathrm{b}$ have been omitted for simplicity, since the formula is valid for both forward and backward incidence directions); that is, the asymmetric transmission can be expressed also through reflection amplitudes. This expression though is based on the fact that $|\mathbf{R}|^{2}+|\mathbf{T}|^{2}=1$, and it is not valid in the presence of absorption. However, for small absorption, a significant difference in the reflection amplitudes $\left|R_{x x}\right|$ and $\left|R_{y y}\right|$, as in the case of Figure 2 in the frequency region around $21.5 \mathrm{THz}$, can be considered as a signature of asymmetric transmission. (Note that the equality of the cross-polarized reflection amplitudes I $R_{x y} \mid$ and $\left|R_{y x}\right|$, which was assumed above, is a direct result of reciprocity and the structure symmetry. ${ }^{27}$ )

Asymmetric Transmission Origin. To investigate the origin of the AT band at $\sim 21.5 \mathrm{THz}$, we examined the field distribution in this spectral region. An example is shown in Figure $5 b$, where we plot the magnetic field amplitude at 21.5 $\mathrm{THz}$ for the incident field configuration shown in Figure 5a. The dominant magnetic field direction in the SCRs is indicated with arrows in Figure 5b.

Observing the fields (both amplitudes and phases) for the configuration of Figure 5a (i.e., for an $x$-polarized incident field) one can see that below $21 \mathrm{THz}$ the structure behaves as a total
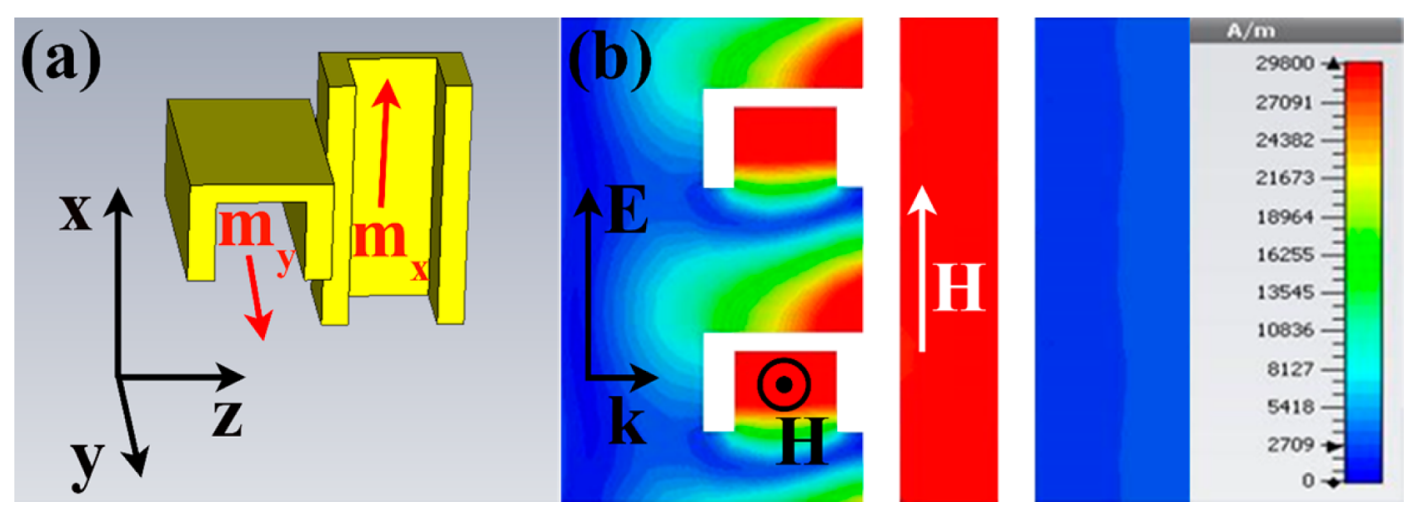

Figure 5. (a) Unit cell of the 3D SCR metamaterials with the incident EM field considered. $\mathbf{m}_{x}$ and $\mathbf{m}_{y}$ are the resonant magnetic moments induced in the SCRs. (b) Total magnetic field amplitude in two unit cells (along the $\mathbf{E}$ direction) of the structure under consideration. The dominant direction of this field is shown with arrows. 
reflector, with its response dominated by the metallic cubes parallel to the electric field, which behave as continuous wires. ${ }^{28,29}$ Approaching $21 \mathrm{THz}$, a strong magnetic field $H_{y}$ is induced in the parallel to the incident magnetic field SCRs, which induces a strong magnetic field $H_{x}$ in the second (perpendicular) SCRs (due to the physical connection, i.e., the conductive coupling, of the two SCRs), giving rise to a $y$ polarized transmitted wave. In other words, the incident magnetic field (along the $y$-direction) excites circulating currents, creating a resonant magnetic dipole moment $\mathbf{m}_{y}$ in the first SCR; these currents, due to the physical connection of the two SCRs, lead to a potential difference between the two parallel to $x-y$ plane sides of the second SCR, and this difference is compensated by the circulating current, creating a magnetic moment $\mathbf{m}_{x}$ at the second SCR and thus providing cross-polarization conversion. This coupling is different in magnitude if the wave propagates toward the opposite (negative $z$ ) direction (note that in the opposite direction to excite the parallel to the incident magnetic field cubes, the incident wave has to "penetrate" a grid of metallic cubes parallel to its electric field; also the generated cross-polarized wave in order to be transmitted needs to "penetrate" a metal cube grid parallel to its electric field; the result is much smaller transmitted intensity). The magnetic resonance origin of the transmission at $\sim 21.5 \mathrm{THz}$ is confirmed also from the fact that the $21.5 \mathrm{THz}$ frequency almost coincides with the magnetic resonance frequency of a single SCR.

The asymmetric transmission band at $\sim 21.5 \mathrm{THz}$ implies a combination of magnetic resonance with electric response of wire-like systems, making our SCR system analogous to combined split-ring resonator and wires systems, extensively discussed in previous literature. ${ }^{29}$ This analogy suggests some important capabilities of our SCR system: ${ }^{29}$ (a) by modifying the cross-section of the SCRs and/or the size of the unit cell one can greatly tune the structure impedance, matching that to the impedance of free space and achieving the highest possible asymmetric transmission through the structure; (b) by modifying the structure one can combine electric and magnetic responses in such a way as to achieve backward propagation in the asymmetric transmission band, thus realizing one-way backward asymmetric transmission components.

Polarization Transformer Response. Analyzing further the response of our structure at the AT band at $\sim 21.5 \mathrm{THz}$ by examining the transmission matrix components shown in Figure $3 \mathrm{a}$, one can see that an $x$-polarized incident wave is almost totally transformed to a $y$-polarized wave when passing through the structure. This indicates a close to $90^{\circ}$ "one-way" optical activity of the structure. To confirm this response, we calculated the ellipticity and the optical activity for an $x$ polarized incident wave transmitted through the structure along the positive $z$-direction. For the calculation, we followed the approach of ref 29; the details are discussed in the Supporting Information. The results are shown in Figure 6, indicating at the asymmetric transmission band more than $90^{\circ}$ pure optical activity, i.e., optical activity associated with close to zero ellipticity of the transmitted wave. This validates the potential of our structure to be used as a $90^{\circ}$ one-way polarization converter.

\section{NANOMETER-SCALE SCR STRUCTURE}

The asymmetric transmission and the resulting polarization control capabilities of the SCR design proposed here prompted us to examine the possibility to apply and exploit the same

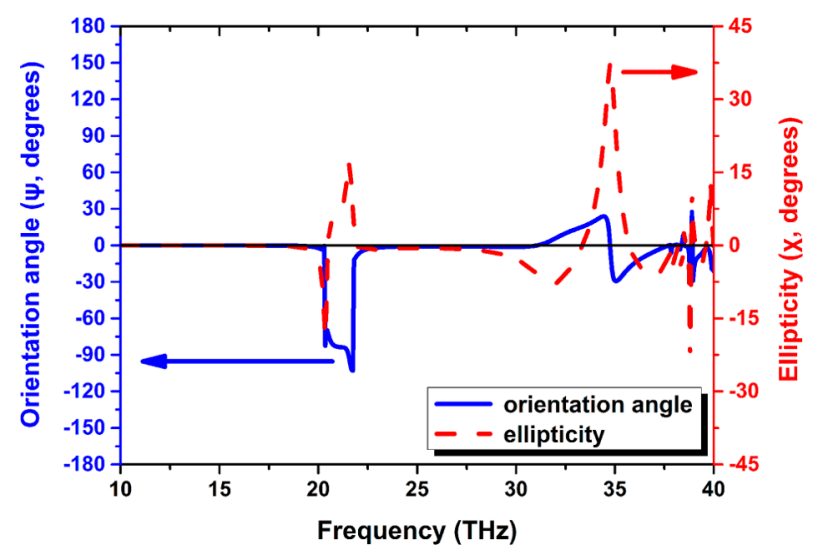

Figure 6. Simulated orientation angle, $\psi$ (blue solid line), and ellipticity angle, $\chi$ (red dashed line), of the polarization ellipse for the wave transmitted through the $3 \mathrm{D}$ SCR metamaterials under consideration, when the metamaterial is excited by an $x$-polarized incident wave propagating along the forward $z$-direction.

design also in the optical region. As has been shown quite recently though, ${ }^{30,31}$ the high losses and the large kinetic inductance of the metals in the optical region result in nonscalability of the magnetic resonance response of magnetic metamaterials with the structure size; in particular they lead to weakening and frequency saturation of the magnetic resonance in nanoscale magnetic metamaterials as the present SCR structure. Despite that, as we show here, the asymmetric transmission properties and capabilities of the proposed design can also be achieved and exploited in the optical region. The weakening of the magnetic response and the modification of the electric response of the structure (due to the change in the metal response from IR to visible) result in, besides higher losses, alteration of the structure impedance, making it better matched to the free-space impedance and thus beneficial for the AT response.

By scaling down the design of Figure 1 uniformly by a factor of 100 and calculating the asymmetric transmission response of the resulting structure one achieves the results shown in Figure 7. Figure 7a shows the transmission amplitudes for a linearly polarized incident wave polarized along the $x$ - and $y$-direction, while Figure $7 \mathrm{~b}$ shows the asymmetric transmission parameter $\Delta$, obtained through eq 4 . Observing the results of Figure 7, one can see that the asymmetric transmission here is even larger than that of a similar infrared structure (compare Figure $7 \mathrm{~b}$ with Figure 4) despite the larger losses of the metal in the optical region. This larger transmission is a result of the abovementioned better impedance match of the structure with its environment, associated with smaller reflectance. (A related slight disadvantage is the larger copolarized transmittance, which degrades the "zero" vs "large" transmittance picture, which we discussed in Section 5.2.) The results of Figure 7 show the possibility to transfer and exploit our structure potential and functionalities also in the optical region, despite the non-size-scalability of the magnetic metamaterial response up to this region (implying also that the strength of the magnetic response is not always essential for significant asymmetric transmission achievement).

\section{CONCLUSIONS}

We studied theoretically and experimentally the asymmetric transmission properties and the polarization control capabilities 

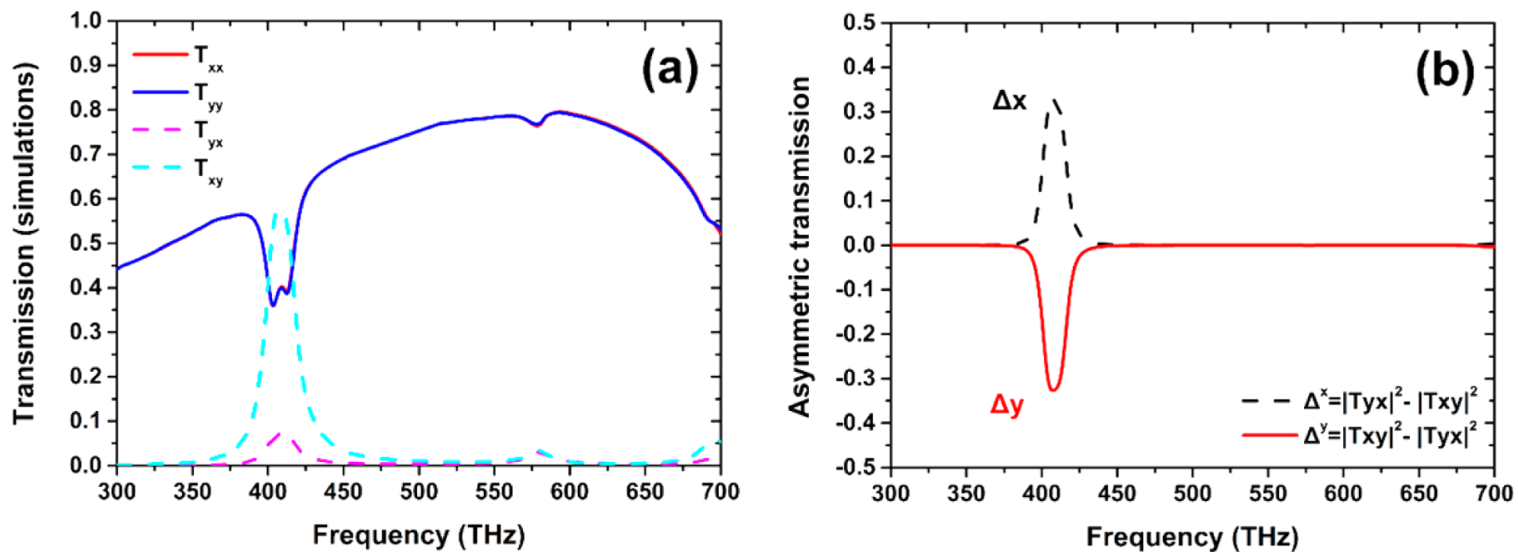

Figure 7. Simulated magnitude of the linearly polarized transmission components (a) and simulated asymmetric transmission parameter $\Delta$ (b) for the 3D SCR metamaterial shown in Figure 1 scaled down uniformly by a factor of 100. The silver in this case is simulated using the data by P. B. Johnson and R. W. Cristy. ${ }^{32}$

of a novel 3D infrared metamaterial structure obtained by employing direct laser writing and selective silver coating. The structure is composed of two layers of split-cube resonators, mutually twisted by $90^{\circ}$. The experimental study was done through reflection measurements, while associated transmission and reflection simulations revealed quite large asymmetric transmission for linearly polarized waves, zero versus quite large transmittance if the structure is "seen" from two opposite directions, and $90^{\circ}$ one-way pure optical activity. The achievement of these nice asymmetric-transmission-related properties of the structure is due to the combination of both electric and magnetic responses of the SCRs and offers additionally the possibility of impedance control (adjusting thus the asymmetric transmission values) and of "one-way" backward propagation. Finally we showed that the nice asymmetric transmission properties of the structure can be transferred and exploited also in the optical region by uniformly scaling the structure unit cell.

In summary, the present study showed that this type of three-dimensional asymmetric transmission structure like the one discussed here, which involves both magnetic and electric resonators, offers unique capabilities in polarization control, something that makes them significant candidates for polarization manipulation components in optics.

\section{ASSOCIATED CONTENT}

\section{S Supporting Information}

Detailed information about the structure fabrication, structural characterization, and polarization transformer response. This material is available free of charge via the Internet at http:// pubs.acs.org.

\section{AUTHOR INFORMATION}

\section{Corresponding Author}

*E-mail: gkenanak@iesl.forth.gr.

\section{Notes}

The authors declare no competing financial interest.

\section{ACKNOWLEDGMENTS}

This work was supported by Greek GSRT project ERC02EXEL and the 3DSET THALIS Program of the Hellenic Ministry of Education (MIS380278). Work at Ames Laboratory was partially supported by the Department of Energy (Basic
Energy Sciences, Division of Materials Sciences and Engineering) under Contract No. DE-AC02-07CH11358 (computational studies).

\section{REFERENCES}

(1) Veselago, V. G. The electrodynamics of substances with simultaneously negative values of $\varepsilon$ and $\mu$. Sov. Phys. Usp. 1968, 10, 509-514.

(2) Smith, D. R.; Pendry, J. B.; Wiltshire, M. C. K. Metamaterials and negative refractive index. Science 2004, 305, 788-792.

(3) Soukoulis, C. M.; Wegener, M. Past achievements and future challenges in the development of three-dimensional photonic metamaterials. Nat. Photonics 2011, 5, 523-530.

(4) Gansel, J. K.; Thiel, M.; Rill, M. S.; Decker, M.; Bade, K.; Saile, V.; von Freymann, G.; Linden, S.; Wegener, M. Gold helix photonic metamaterial as broadband circular polarizer. Science 2009, 325, 15131515.

(5) Kabashin, A. V.; Evans, P.; Pastkovsky, S.; Hendren, W.; Wurtz, G. A.; Atkinson, R.; Pollard, R.; Podolskiy, V. A.; Zayats, A. V. Plasmonic nanorod metamaterials for biosensing. Nat. Mater. 2009, 8, $867-871$.

(6) Leung, D. M. H.; Rahman, B. M. A.; Grattan, K. T. V. Numerical analysis of asymmetric silicon nanowire waveguide as compact polarization rotator. IEEE Photonics J. 2011, 3, 381-389.

(7) Hogan, C. L. The ferromagnetic faraday effect at microwave frequencies and its applications. Rev. Mod. Phys. 1953, 25, 253-263.

(8) Fedotov, V. A.; Mladyonov, P. L.; Prosvirnin, S. L.; Rogacheva, A. V.; Chen, Y.; Zheludev, N. I. Asymmetric propagation of electromagnetic waves through a planar chiral structure. Phys. Rev. Lett. 2006, 97 (16), 167401.

(9) Plum, E.; Fedotov, V. A.; Zheludev, N. I. Planar metamaterial with transmission and reflection that depend on the direction of incidence. Appl. Phys. Lett. 2009, 94, 131901.

(10) Menzel, C.; Helgert, C.; Rockstuhl, C.; Kley, E. B.; Tunnermann, A.; Pertsch, T.; Lederer, F. Asymmetric transmission of linearly polarized light at optical metamaterials. Phys. Rev. Lett. 2010, 104, 253902.

(11) Singh, R.; Plum, E.; Menzel, C.; Rockstuhl, C.; Azad, A. K.; Cheville, R. A.; Lederer, F.; Zhang, W.; Zheludev, N. I. Terahertz metamaterial with asymmetric transmission. Phys. Rev. B 2009, 80, 153104.

(12) Schwanecke, A. S.; Fedotov, V. A.; Khardikov, V. V.; Prosvirnin, S. L.; Chen, Y.; Zheludev, N. I. Nanostructured metal film with asymmetric optical transmission. Nano Lett. 2008, 8, 2940-2943.

(13) Drezet, A.; Genet, C.; Laluet, J. Y.; Ebbesen, T. W. Optical chirality without optical activity: how surface plasmons give a twist to light. Opt. Express 2008, 16, 12559-12570. 
(14) Plum, E.; Fedotov, V. A.; Zheludev, N. I. Asymmetric transmission: a generic property of two-dimensional periodic patterns. J. Opt. 2011, 13, 024006.

(15) Menzel, C.; Rockstuhl, C.; Lederer, F. Advanced Jones calculus for the classification of periodic metamaterials. Phys. Rev. A 2010, 82, 053811 .

(16) Niemi, T.; Karilainen, A. O.; Tretyakov, S. A. Synthesis of polarization transformers. IEEE Trans. Antennas Propag. 2013, 61, $3102-3111$.

(17) Kang, M.; Chen, J.; Cui, H. X.; Li, Y. N.; Wang, H. T. Asymmetric transmission for linearly polarized electromagnetic radiation. Opt. Express 2011, 19, 8347-8356.

(18) Mutlu, M.; Akosman, A. E.; Serebryannikov, A. E.; Ozbay, E. Asymmetric transmission of linearly polarized waves and polarization angle dependent wave rotation using a chiral metamaterial. Opt. Express 2011, 19, 14290-14299.

(19) Huang, C.; Feng, Y. J.; Zhao, J. M.; Wang, Z. B.; Jiang, T. Asymmetric electromagnetic wave transmission of linear polarization via polarization conversion through chiral metamaterial structures. Phys. Rev. B 2012, 85, 195131.

(20) Malinauskas, M.; Farsari, M.; Piskarskas, A.; Juodkazis, S. Ultrafast laser nanostructuring of photopolymers: a decade of advances. Phys. Rep. 2013, 533, 1-31.

(21) Farsari, M.; Chichkov, B. N. Two-photon fabrication. Nat. Photonics 2009, 3, 450-452.

(22) Vasilantonakis, N.; Terzaki, K.; Sakellari, I.; Purlys, V.; Gray, D.; Soukoulis, C. M.; Vamvakaki, M.; Kafesaki, M.; Farsari, M. Threedimensional metallic photonic crystals with optical bandgaps. Adv. Mater. 2012, 24, 1101-1105.

(23) Terzaki, K.; Vasilantonakis, N.; Gaidukeviciute, A.; Reinhardt, C.; Fotakis, C.; Vamvakaki, M.; Farsari, M. 3d conducting nanostructures fabricated using direct laser writing. Opt. Mater. Express 2011, 1, 586-597.

(24) Zhou, J. F.; Chowdhury, D. R.; Zhao, R. K.; Azad, A. K.; Chen, H. T.; Soukoulis, C. M.; Taylor, A. J.; O’Hara, J. F. Terahertz chiral metamaterials with giant and dynamically tunable optical activity. Phys. Rev. B 2012, 86, 035448.

(25) Wang, B. N.; Zhou, J. F.; Koschny, T.; Soukoulis, C. M. Nonplanar chiral metamaterials with negative index. Appl. Phys. Lett. 2009, 94, 151112.

(26) Li, Z. F.; Mutlu, M.; Ozbay, E. Highly asymmetric transmission of linearly polarized waves realized with a multilayered structure including chiral metamaterials. J. Phys. D: Appl. Phys. 2014, 47, 075107.

(27) Mazneva, A. A.; Every, A. G.; Wrightc, O. B. Reciprocity in reflection and transmission: What is a 'phonon diode'? Wave Motion 2013, 50, 776-784.

(28) Pendry, J.; Holden, A.; Stewart, W.; Youngs, I. Extremely low frequency plasmons in metallic mesostructures. Phys. Rev. Lett. 1996, 76, 4773.

(29) Bassiri, S.; Papas, C. H.; Engheta, N. Electromagnetic wave propagation through a dielectric-chiral interface and through a chiral slab. J. Opt. Soc. Am. A 1988, 5, 1450-1459.

(30) Zhou, J.; Koschny, Th.; Kafesaki, M.; Economou, E. N.; Pendry, J. B.; Soukoulis, C. M. Saturation of the magnetic response of split-ring resonators at optical frequencies. Phys. Rev. Lett. 2005, 95, 223902.

(31) Penciu, R. S.; Kafesaki, M.; Koschny, Th.; Economou, E. N.; Soukoulis, C. M. Magnetic response of nanoscale left-handed metamaterials. Phys. Rev. B 2010, 81, 235111.

(32) Johnson, P. B.; Christy, R. W. Optical constants of the noble metals. Phys. Rev. B 1972, 6, 4370. 